\title{
On the Chvátal-Erdős triangle game
}

\author{
József Balogh* and Wojciech Samotij ${ }^{\dagger}$
}

Submitted: Oct 13, 2010; Accepted: Mar 22, 2011; Published: Mar 31, 2011

Mathematics Subject Classification: 05C57, 05C35, 91A24, 91A43, 91A46

\begin{abstract}
Given a graph $G$ and positive integers $n$ and $q$, let $\mathbf{G}(G ; n, q)$ be the game played on the edges of the complete graph $K_{n}$ in which the two players, Maker and Breaker, alternately claim 1 and $q$ edges, respectively. Maker's goal is to occupy all edges in some copy of $G$; Breaker tries to prevent it. In their seminal paper on positional games, Chvátal and Erdős proved that in the game $\mathbf{G}\left(K_{3} ; n, q\right)$, Maker has a winning strategy if $q<\sqrt{2 n+2}-5 / 2$, and if $q \geq 2 \sqrt{n}$, then Breaker has a winning strategy. In this note, we improve the latter of these bounds by describing a randomized strategy that allows Breaker to win the game $\mathbf{G}\left(K_{3} ; n, q\right)$ whenever $q \geq(2-1 / 24) \sqrt{n}$. Moreover, we provide additional evidence supporting the belief that this bound can be further improved to $(\sqrt{2}+o(1)) \sqrt{n}$.
\end{abstract}

\section{Introduction}

In a positional game, the two players, traditionally called Maker and Breaker, alternately occupy previously unoccupied elements of a given finite set $X$. Maker wins if he manages to completely occupy one of the members of a prescribed set system $\mathcal{H} \subseteq 2^{X}$, otherwise Breaker wins. A particular family of positional games originates from a seminal paper of Chvátal and Erdős [4]. Let $\mathcal{P}$ be a monotone graph property. In the biased $\mathcal{P}$-game Maker and Breaker are alternately claiming 1 and at most $q$ edges of the complete graph $K_{n}$ per round, respectively. Maker's goal is to build a graph with property $\mathcal{P}$; Breaker wins the game if he prevents Maker from achieving this goal after all $\left(\begin{array}{l}n \\ 2\end{array}\right)$ edges of $K_{n}$ have been occupied. Chvátal and Erdös [4] asked about the threshold for the bias $q$ in such a

*Department of Mathematics, University of Illinois, 1409 W Green Street, Urbana, IL 61801, USA; and Department of Mathematics, University of California, San Diego, 9500 Gilman Drive, La Jolla, CA 92093, USA. E-mail address: jobal@math.uiuc.edu. This material is based upon work supported by NSF CAREER Grant DMS-0745185, UIUC Campus Research Board Grants 09072 and 08086, and OTKA Grant K76099.

${ }^{\dagger}$ Department of Mathematics, University of Illinois, Urbana, IL, 61801, USA. E-mail address: wojteksa@gmail.com. 
game, i.e., the value of $q_{0}(n)$ such that Maker has a winning strategy if $q \leq q_{0}(n)$ and the game is a Breaker's win if $q>q_{0}(n)$.

In [4], three particular games of this type are analyzed. In the connectivity game, Maker tries to occupy all edges of some spanning tree. Chvátal and Erdős [4] showed that Breaker wins the connectivity game when $q \geq(1+o(1)) n / \log n$. This was proved to be asymptotically optimal by Gebauer and Szabó [5], who provided a strategy for Maker that allows him to win the connectivity game when $q \leq(1-o(1)) n / \log n$. In the Hamiltonicity game, Maker tries to occupy all edges of some Hamilton cycle. In [4], it is shown that Breaker wins the Hamiltonicity game when $q \geq(1+o(1)) n / \log n$. This was again proved to be asymptotically optimal by Krivelevich [8], who found a strategy for Maker that allows him to win the Hamiltonicity game when $q \leq(1-o(1)) n / \log n$. In the subgraph game, which is the main subject of this note, Maker tries to occupy all edges of some copy of a fixed graph. Other positional games on graphs that have attracted considerable attention include the diameter game [1], the planarity, colorability, and minor games [6]. For more information on positional games, we refer the reader to the recent monograph of Beck [2].

For a graph $G$ and positive integers $n$ and $q$, we let $\mathbf{G}(G ; n, q)$ be the game on the edge set of the complete graph $K_{n}$ in which Maker and Breaker alternately claim 1 and $q$ edges, respectively. Maker's goal is to claim all edges in some copy of $G$ and Breaker tries to prevent it. In the special case $G=K_{3}$, the following theorem was proved in [4].

Theorem 1. If $q<\sqrt{2 n+2}-5 / 2$, then Maker has a winning strategy for $\mathbf{G}\left(K_{3} ; n, q\right)$. On the other hand, if $q \geq 2 \sqrt{n}$, then Breaker has a winning strategy for $\mathbf{G}\left(K_{3} ; n, q\right)$.

Bednarska and Luczak [3] obtained the following wide generalization of Theorem 1.

Theorem 2. For every graph $G$ that contains at least 3 non-isolated vertices, there exist positive constants $c_{0}, C_{0}$, and $n_{0}$ such that for every $n$ with $n \geq n_{0}$, the following holds.

1. If $q \leq c_{0} n^{1 / m(G)}$, then Maker has a winning strategy in the game $\mathbf{G}(G ; n, q)$.

2. If $q \geq C_{0} n^{1 / m(G)}$, then Breaker has a winning strategy in the game $\mathbf{G}(G ; n, q)$.

In the above,

$$
m(G)=\max \left\{\frac{e(H)-1}{v(H)-2}: H \subseteq G \text { with } v(H) \geq 3\right\} .
$$

They also conjectured that the constants $c_{0}$ and $C_{0}$ in Theorem 2 can be chosen arbitrarily close to each other.

Conjecture 3. For every graph $G$ and positive constant $\varepsilon$, there exist a positive constant $c$ and a natural number $n_{0}$ such that for every $n$ with $n \geq n_{0}$, the following holds.

1. If $q \leq(c-\varepsilon) n^{1 / m(G)}$, then Maker has a winning strategy in the game $\mathbf{G}(G ; n, q)$.

2. If $q \geq(c+\varepsilon) n^{1 / m(G)}$, then Breaker has a winning strategy in the game $\mathbf{G}(G ; n, q)$. 
In contrast to the recent results on the connectivity and Hamiltonicity games, so far there has been no progress towards resolving Conjecture 3. In fact, there is no non-trivial graph $G$, for which the existence of a constant $c$ as above has been proved or disproved. In this note, in an attempt to resolve Conjecture 3, we consider the simplest non-trivial case $G=K_{3}$. Our main result is the following strengthening of Theorem 1 .

Theorem 4. If $q \geq(2-1 / 24) \sqrt{n}$, then for almost all $n$, Breaker has a winning strategy in the game $\mathbf{G}\left(K_{3} ; n, q\right)$.

Remark. A more careful analysis of Breaker's strategy described in the proof of Theorem 4 shows that Breaker can win the game $\mathbf{G}\left(K_{3} ; n, q\right)$ even under the slightly weaker assumption that $q \geq 1.935 \sqrt{n}$. Since such refined analysis is significantly more complicated and technical, and the improvement it yields is rather modest, we decided to settle for the weaker bound stated in Theorem 4. For details, we refer the reader to Section 5.

Moreover, to support the belief that the lower bound in Theorem 1 is essentially sharp, we present a strategy for Breaker that allows him to delay his loss until a positive proportion of the edges is occupied, provided that the bias $q$ is larger than $\sqrt{2 n}$.

Theorem 5. For every $\varepsilon \in(0,(2-\sqrt{2}) / 3)$, if $q \geq(\sqrt{2}+3 \varepsilon) \sqrt{n}$, then in the game $\mathbf{G}\left(K_{3} ; n, q\right)$, Breaker has a strategy that prevents Maker from winning in the first $\varepsilon^{3} n^{3 / 2} / 8$ rounds.

Both bounds in Theorem 1 are quite easy to prove. For the lower bound, if $q<$ $\sqrt{2 n+2}-5 / 2$, then there is a simple winning strategy for Maker. He chooses a vertex $u$ and claims only edges incident to $u$. Regardless of how Breaker plays, after at most about $\sqrt{2 n}$ rounds, there will be some $v$ and $w$ such that $u v$ and $u w$ were claimed by Maker and $v w$ is available. Hence, Maker can close the triangle uvw and win the game quickly. For the upper bound, assume that $q \geq 2 \sqrt{n}$. Whenever Maker claims an edge $u v$, Breaker responds with $q / 2$ edges at both $u$ and $v$, making sure that he 'closes' all paths of length 2 in Maker's graph. More precisely, Breaker claims his $q$ edges in such a way that after his move there is no $z$ such that $u z$ belongs to Maker's graph and $v z$ is unclaimed or vice versa. This is possible since the maximum degree in Maker's graph will never exceed $2 n / q$, and $2 n / q \leq q / 2$.

Breaker's strategy described in the proof of Theorem 4 is a refinement of the above. We now allow Breaker to claim edges randomly, causing them to be more uniformly distributed. One would expect that as a result, every time Maker joins two high-degree vertices, many new paths of length 2 that appear in his graph are already 'closed' with Breaker's random edges and consequently, Breaker can allow the degrees in Maker's graph to grow somewhat larger than $q / 2$, which in turn relaxes the bound on $q$. Unfortunately, our life is not that easy. Breaker has to immediately 'close' all paths of length 2 that appear in Maker's graph after Maker claims some edge $u v$. If the degree of $u$ in Maker's graph is high, then Breaker can claim only very few edges incident to $v$ at random. In order to overcome this problem, we further refine Breaker's strategy by allowing him to claim different number of edges incident to $u$ and $v$, depending on their degrees is Maker's 
graph. As a consequence, every vertex with high degree in Maker's graph is either incident to many 'random' Breaker's edges or its degree in Breaker's graph is unusually high. Although this idea is quite natural, its details are rather delicate and the analysis of the game becomes quite involved.

\section{A concentration result}

Let $m, n$, and $N$ be positive integers satisfying $m, n \leq N$. A random variable $X$ has hypergeometric distribution with parameters $N, m$, and $n$, denoted $\operatorname{Hypergeometric}(N, m, n)$, if it describes the number of successes in a sequence of $n$ draws from a set of size $N$ with $m$ marked elements, without replacement. In other words, $X$ is the integer-valued random variable satisfying

$$
P(X=k)=\frac{\left(\begin{array}{c}
m \\
k
\end{array}\right)\left(\begin{array}{c}
N-m \\
n-k
\end{array}\right)}{\left(\begin{array}{c}
N \\
n
\end{array}\right)} \text { for all } k \in\{0, \ldots, m\} .
$$

We will need the following standard estimate on the tail probabilities of hypergeometric random variables, see [7, Section 6].

Lemma 6. Let $N, m$, and $n$ be positive integers with $m, n \leq N$ and $m \leq N / 2$. Let $X$ be a hypergeometric random variable with parameters $N, m$, and $n$, and let $\mu=\mathbb{E}[X]=m n / N$. If $t \geq 0$, then

$$
P(X \leq \mu-t) \leq e^{-t^{2} /(2 \mu)}
$$

\section{Proof of Theorem 5}

As Maker and Breaker claim their edges during the game, they are building two edgedisjoint subgraphs of $K_{n}$, denoted by $G_{M}$ and $G_{B}$, respectively. We will denote the number of edges in these subgraphs by $e_{M}$ and $e_{B}$, respectively. Finally, given a vertex $v, N_{M}(v)$ will denote the neighborhood of $v$ in the graph $G_{M}$, and $\operatorname{deg}_{M}(v), \operatorname{deg}_{B}(v)$ will denote the degrees of $v$ in $G_{M}$ and $G_{B}$, respectively.

Assume that $q \geq(\sqrt{2}+3 \varepsilon) \sqrt{n}$ for some $\varepsilon \in(0,(2-\sqrt{2}) / 3)$. Below we describe a strategy for Breaker that prevents Maker from winning in the first $\left(\varepsilon^{3} / 8\right) n^{3 / 2}$ rounds. At all times during the game, Breaker will keep track of the following two sets of vertices of $K_{n}$ :

$$
A=\left\{v: \operatorname{deg}_{M}(v) \geq \varepsilon \sqrt{n} / 2\right\}, \quad \text { and } \quad B=\left\{v: \operatorname{deg}_{M}(v) \geq \varepsilon \sqrt{n}\right\} .
$$

For two vertices $u$ and $v$, we write $u \leq_{A} v$ if $u$ entered $A$ earlier than $v$. Note that at all times $\leq_{A}$ is a linear order on $A$. The strategy that we describe will allow Breaker to reach the following goal.

Goal. Suppose that $e_{M} \leq\left(\varepsilon^{3} / 8\right) n^{3 / 2}$. Then the following holds.

I. At the end of each round, after Breaker's move, for every vertex $v, N_{M}(v)$ is a clique in $G_{B}$. 
II. The maximum degree of $G_{M}$ is at most $(\sqrt{2}+\varepsilon) n^{1 / 2}$.

III. If $u, v \in B$, then $u v \in G_{M} \cup G_{B}$.

Note that achieving Goal I guarantees that Maker will not claim all three edges of some triangle, since every time it is his turn to move, there is a Breaker's edge in every triangle in which Maker has managed to claim two edges.

We describe the strategy for Breaker. Suppose that Maker plays an edge at a vertex $u \notin B$. Then Breaker will use at most $\varepsilon \sqrt{n}$ edges to attach $u$ to the $\varepsilon \sqrt{n}$ smallest (with respect to the ordering $\leq_{A}$ ) of its non-neighbors in $G_{M} \cup G_{B}$. Clearly, he can do it while claiming at most $2 \varepsilon \sqrt{n}$ edges per round (at most $\varepsilon \sqrt{n}$ edges for each endpoint of the edge claimed by Maker). We start by showing that this guarantees that Goal III is reached. Note first that if $e_{M} \leq\left(\varepsilon^{3} / 8\right) n^{3 / 2}$, then

$$
|A| \leq 2 e_{M} /(\varepsilon \sqrt{n} / 2) \leq\left(\varepsilon^{2} / 2\right) n
$$

Fix some $u, v \in B$. Since $B \subseteq A$, then $u$ and $v$ are $\leq_{A}$-comparable, and therefore we may assume that $u \leq_{A} v$. It follows that from the moment when $u$ entered $A$ until the moment when $v$ entered $B$, Maker claimed more than $(\varepsilon / 2) \sqrt{n}$ edges at $v$. Therefore, Breaker had the opportunity to guarantee that $v$ is adjacent to at least $(\varepsilon / 2) \sqrt{n} \cdot \varepsilon \sqrt{n}$ elements of $A$ that entered $A$ before $v$. In particular, since $u$ entered $A$ before $v,(2)$ implies that when $v$ enters $B, v$ is already adjacent to $u$.

Suppose that at the beginning of some round, Goals I and II are reached, and Maker plays an edge $u v$. We have already shown that $\{u, v\} \nsubseteq B$, hence it suffices to consider the two remaining cases.

Case 1. $u, v \notin B$.

Breaker claims all vacant edges from $u$ to $N_{M}(v)$ and all vacant edges from $v$ to $N_{M}(u)$. Since $u, v \notin B$, he needs at most $2 \varepsilon \sqrt{n}$ edges, apart from the at most $2 \varepsilon \sqrt{n}$ edges which Breaker claimed to join $u$ and $v$ to their $\leq_{A}$-smallest non-neighbors in $A$. By our assumptions on $\varepsilon$ and $q$, Breaker uses at most $q$ edges.

Case 2. $u \notin B, v \in B$.

Breaker claims all vacant edges from $u$ to $N_{M}(v)$ and all vacant edges from $v$ to $N_{M}(u)$. Apart from these, he also claims at most $\varepsilon \sqrt{n}$ edges joining $u$ to its $\leq_{A}$-smallest non-neighbors in $A$. This is possible, since by our assumptions,

$$
\operatorname{deg}_{M}(v)+\operatorname{deg}_{M}(u)+\varepsilon \sqrt{n} \leq \Delta\left(G_{M}\right)+2 \varepsilon \sqrt{n} \leq q
$$

Finally, Breaker uses his remaining moves to claim arbitrary edges incident to $v$, which gives the total of at least $(\sqrt{2}+2 \varepsilon) \sqrt{n}-\operatorname{deg}_{M}(v)$ edges claimed at $v$.

Clearly, since Breaker 'closed' all new paths of length 2 that appeared in $G_{M}$ after Maker's move, Goal I continues to be satisfied. It remains to prove that Goal II is also satisfied. Suppose that at the end of some round, the degree of some vertex $v$ in $G_{M}$ exceeds $(\sqrt{2}+\varepsilon) \sqrt{n}$. Since every time Maker increased the degree of $v$ in $G_{M}$ from some 
$d-1$ to $d$, where $d \geq \varepsilon \sqrt{n}$, that is, $v$ was in $B$, Breaker claimed at least $(\sqrt{2}+2 \varepsilon \sqrt{n})-d$ edges at $v$, it follows that

$$
\operatorname{deg}_{B}(v) \geq \sum_{d=\varepsilon \sqrt{n}}^{(\sqrt{2}+\varepsilon) \sqrt{n}}((\sqrt{2}+2 \varepsilon) \sqrt{n}-d) \geq \sqrt{2 n} \cdot \frac{(\sqrt{2}+\varepsilon) \sqrt{n}+\varepsilon \sqrt{n}}{2}>n
$$

which is a clear contradiction.

\section{Proof of Theorem 4}

As Maker and Breaker claim their edges during the game, they are building two edgedisjoint subgraphs of $K_{n}$, denoted by $G_{M}$ and $G_{B}$, respectively. More precisely, the edges that Maker picks in the first $t$ rounds form the graph $G_{M}^{(t)}$, and the edges that Breaker claims in the first $t$ rounds form the graph $G_{B}^{(t)}$. Given a vertex $v$, we will denote its degrees in these subgraphs by $\operatorname{deg}_{M}^{(t)}(v)$ and $\operatorname{deg}_{B}^{(t)}(v)$. The neighborhoods of $v$ in $G_{M}^{(t)}$ and $G_{B}^{(t)}$ will be denoted by $N_{M}^{(t)}(v)$ and $N_{B}^{(t)}(v)$, respectively. An edge $e \in E\left(K_{n}\right)$ is claimed if $e \in G_{M} \cup G_{B}$; otherwise, $e$ is unclaimed or vacant.

We start with a few definitions. Let

$$
q=\left(2-\frac{1}{24}\right) \sqrt{n}, \quad \alpha=\frac{\sqrt{n}}{7}, \quad \beta=\frac{3 \sqrt{n}}{4}, \quad \text { and } \quad \Delta=\frac{2 n-\alpha \beta}{q-\alpha} \approx 1.043 \sqrt{n}
$$

A vertex $v$ will be called large at time $t$ if $\operatorname{deg}_{M}^{(t)}(v)>\beta$; otherwise $v$ will be called small. When $v$ is a large vertex, we let

$$
\alpha_{v}^{(t)}=\frac{\operatorname{deg}_{M}^{(t)}(v)-\beta}{\Delta-\beta} \cdot \alpha
$$

Finally, we would like to warn the reader that for the sake of clarity of the presentation, we will sometimes omit the superscript $(t)$ in $G_{B}^{(t)}, \operatorname{deg}_{M}^{(t)}, \alpha_{v}^{(t)}$, etc.

Claim 7. For all large $v$ with $\operatorname{deg}_{M}(v) \leq \Delta$, we have $\alpha_{v} \in[0, \alpha]$ and

$$
q / 2+\alpha_{v}-\operatorname{deg}_{M}(v)=q / 2+\alpha-\Delta+\left(1-\frac{\alpha_{v}}{\alpha}\right)(\Delta-\alpha-\beta) \in[0, q / 2-\beta] .
$$

Proof. The assumption on $v$ immediately implies that $\alpha_{v} \in[0, \alpha]$. In order to see that the equality in (3) holds, note that both sides are linear in $\operatorname{deg}_{M}(v)$, and equality holds when $\operatorname{deg}_{M}(v)=\beta$ (since then $\left.\alpha_{v}=0\right)$ and when $\operatorname{deg}_{M}(v)=\Delta\left(\right.$ since then $\left.\alpha_{v}=\alpha\right)$. The inequality $q / 2+\alpha_{v}-\operatorname{deg}_{M}(v) \geq 0$ holds because $q / 2+\alpha-\Delta \geq 0,\left(1-\alpha_{v} / \alpha\right) \geq 0$, and $\Delta-\alpha-\beta \geq 0$. Finally, $q / 2+\alpha_{v}-\operatorname{deg}_{M}(v) \leq q / 2-\beta$ because $\left(1-\alpha_{v} / \alpha\right) \leq 1$ and $(\Delta-\alpha-\beta) \geq 0$. 
We describe the strategy for Breaker. Breaker's main goal will be to kill all immediate threats, i.e., to make sure that for all $t$ and every vertex $v$, at the end of round $t$, the set $N_{M}^{(t)}(v)$ is a clique in $G_{B}^{(t)}$. If Breaker manages to achieve this goal, this will clearly mean that Maker cannot claim three edges in any triangle. Breaker will also make sure that the maximum degree of $G_{M}$ stays below $\Delta$.

Suppose that in some round $t+1$, Maker claims an edge $u v$. Also, assume that $\operatorname{deg}_{M}^{(t)}(u), \operatorname{deg}_{M}^{(t)}(v) \leq \Delta$. There are three cases to consider, depending on the degrees of $u$ and $v$ in $G_{M}$ at the end of round $t$.

Case 1. $u$ and $v$ are small.

Breaker tries to claim $q / 2$ edges incident to each of the two vertices. First, he claims all unclaimed edges from $v$ to $N_{M}^{(t)}(u)$ and all unclaimed edges from $u$ to $N_{M}^{(t)}(v)$. If, immediately after Maker's move, the number of vacant edges at $u$ (or $v$ ) was less than $q / 2$, then Breaker can claim all these edges and not worry about $u$ (or $v$ ) anymore. Otherwise, for each $x \in\{u, v\}$, Breaker randomly claims $q / 2-\beta$ vacant edges at $x$. More precisely, he picks a random ordering of the set of vertices (independently of the current state of the game) and joins $x$ with the first $q / 2-\beta$ vertices $y$ in that ordering such that the edge $x y$ has not been claimed yet. Finally, Breaker uses his remaining moves to arbitrarily claim $\beta-\operatorname{deg}_{M}^{(t)}(u)$ and $\beta-\operatorname{deg}_{M}^{(t)}(v)$ vacant edges at $v$ and $u$, respectively.

Case 2. $u$ is small, $v$ is large.

Breaker tries to claim $q / 2+\alpha_{v}^{(t)}$ edges at $u$ and $q / 2-\alpha_{v}^{(t)}$ edges at $v$. Similarly as in Case 1, without loss of generality we may assume that the number of unclaimed edges at $u$ and $v$ is at least $q / 2+\alpha_{v}^{(t)}$ and $q / 2-\alpha_{v}^{(t)}$, respectively. First, Breaker claims all the unclaimed edges from $v$ to $N_{M}^{(t)}(u)$ and from $u$ to $N_{M}^{(t)}(v)$. This is possible, since by Claim $7, q / 2+\alpha_{v}^{(t)} \geq \operatorname{deg}_{M}^{(t)}(v)$ and $q / 2-\alpha_{v}^{(t)} \geq q / 2-\alpha \geq \beta \geq \operatorname{deg}_{M}^{(t)}(u)$. Next, Breaker randomly claims $q / 2+\alpha_{v}^{(t)}-\operatorname{deg}_{M}^{(t)}(v)$ vacant edges at $u$. Note that by Claim 7 , $q / 2+\alpha_{v}^{(t)}-\operatorname{deg}_{M}^{(t)}(v) \leq q / 2-\beta$. Finally, Breaker claims the remaining $q / 2-\alpha_{v}^{(t)}-\operatorname{deg}_{M}^{(t)}(u)$ edges at $v$ arbitrarily.

Case 3. $u$ and $v$ are large.

Breaker tries to claim all vacant edges from $v$ to $N_{M}^{(t)}(u)$ and from $u$ to $N_{M}^{(t)}(v)$. Since $\operatorname{deg}_{M}^{(t)}(u)+\operatorname{deg}_{M}^{(t)}(v)$ can be greater than $q$, we have to argue that this is possible. It would be possible if

$$
q \geq \operatorname{deg}_{M}^{(t)}(u)+\operatorname{deg}_{M}^{(t)}(v)-e_{B}^{(t)}(u, v),
$$

where $e_{B}^{(t)}(u, v)$ denotes the number of edges in $G_{B}^{(t)}$ between $u$ and $N_{M}^{(t)}(v)$ plus the number of edges in $G_{B}^{(t)}$ between $v$ and $N_{M}^{(t)}(u)$. Assuming that (4) holds, Breaker can clearly claim all such edges, and moreover, he can do it in such a way that he claims at least $\min \left\{q / 2, q-\operatorname{deg}_{M}^{(t)}(u)\right\}$ edges at $u$ and at least $\min \left\{q / 2, q-\operatorname{deg}_{M}^{(t)}(v)\right\}$ edges at $v$. Finally, note that by Claim $7, \min \left\{q / 2, q-\operatorname{deg}_{M}^{(t)}(x)\right\} \geq q / 2-\alpha_{x}^{(t)}$ for all $x \in\{u, v\}$.

Clearly, by following the above strategy, Breaker will 'close' all new paths of length 2 that appear in $G_{M}$ after Maker's move, provided that inequality (4) holds for every $t, u$, and $v$. Hence, Breaker will achieve his main goal - at the end of each round $t$, 
for each vertex $v$, the set $N_{M}^{(t)}(v)$ will be a clique in $G_{B}^{(t)}$. Before we start analyzing inequality (4), we first show that Breaker manages to achieve his secondary goal, i.e., to keep the maximum degree of $G_{M}$ below $\Delta$.

Claim 8. The maximum degree of $G_{M}$ always stays below $\Delta$.

Proof. Suppose that at some point during the game there is a vertex $v$ with $\operatorname{deg}_{M}(v)=\Delta$. Every time Maker claimed an edge incident to $v$, increasing the degree of $v$ in $G_{M}$ from some $d$ to $d+1$, Breaker responded with at least $q / 2$ edges when $d \leq \beta$, or $q / 2-(d-$ $\beta) \alpha /(\Delta-\beta)$ edges when $d>\beta$. It follows that

$$
\operatorname{deg}_{B}(v) \geq \frac{\Delta q}{2}-\sum_{d=\beta+1}^{\Delta-1} \frac{d-\beta}{\Delta-\beta} \alpha \geq \frac{\Delta q}{2}-\frac{(\Delta-\beta) \alpha}{2}=\frac{\Delta(q-\alpha)+\beta \alpha}{2}=n
$$

which is a clear contradiction.

In order to show that the strategy described above prevents Maker from winning, it suffices to prove that whenever Breaker reaches Case 3, inequality (4) will be satisfied. Recall that Breaker employed some randomness to decide how to move. In the remainder of this section, we show that regardless of Maker's strategy, with probability tending to 1 ans $n$ tends to infinity, inequality (4) holds for all $t$ and every pair of vertices $u$ and $v$ that are large at time $t$. Hence, regardless of Maker's strategy, Breaker can win the game $\mathbf{G}\left(K_{3} ; n, q\right)$, provided that $n$ is large enough.

The main reason why one would expect inequality (4) to hold is that by the time the vertices $u$ and $v$ become large, Breaker should claim many random edges incident to both $u$ and $v$. If this was true, it would follow that with high probability $e_{B}(u, v)$ was large enough to make (4) true. Unfortunately, this might not be the case. If Maker consistently joins $u$ and $v$ only to high-degree vertices, it greatly reduces the number of random edges that Breaker can claim at $u$ and $v$. This is the reason why in Case 2, i.e., whenever Maker joins a small vertex to a large vertex, we allow Breaker to claim more edges at the lower-degree vertex. These extra edges drive down the degree in $G_{M}$ of vertices that cannot take full advantage of randomness. The following statement quantifies the above discussion of this randomness versus degree trade-off.

Claim 9. Suppose that a vertex $v$ becomes large at time $t$. Then there exists a $\lambda_{v}^{\prime} \in$ $[0,1]$ such that $\operatorname{deg}_{M}(v)$ never exceeds $\left(2 n-\left(3-2 \lambda_{v}^{\prime}\right) \alpha \beta\right) /(q-\alpha)$ and the total number of random edges that Breaker has claimed at $v$ by the end of round $t$ is at least $\beta\left(q / 2+\alpha-\Delta+\lambda_{v}^{\prime}(\Delta-\alpha-\beta)\right)$. Moreover, $\lambda_{v}^{\prime}$ depends only on what happened in the first $t$ rounds of the game.

Proof. Every time $v$ was small and Maker claimed an edge incident to $v$, there was a $\lambda^{\prime} \in[0,1]$, such that Breaker responded with $q / 2+\alpha-\Delta+\left(1-\lambda^{\prime}\right)(\Delta-\alpha-\beta)$ random edges at $v$ among the total of $q / 2+\lambda^{\prime} \alpha$ edges claimed at $v$. More specifically, $\lambda^{\prime}=0$ whenever Maker claimed an edge between $v$ and another small vertex, and $\lambda^{\prime}=\alpha_{u} / \alpha$ whenever Maker claimed an edge between $v$ and a large vertex $u$. Let $\lambda_{v}^{\prime}$ be the average value of $1-\lambda^{\prime}$ 
over the first $\beta$ edges that Maker claimed at $v$. Clearly, $\lambda_{v}^{\prime} \in[0,1]$. Moreover, it follows from the definition of $\lambda_{v}^{\prime}$ that Breaker claimed at least $\beta\left(q / 2+\alpha-\Delta+\lambda_{v}^{\prime}(\Delta-\alpha-\beta)\right)$ random edges at $v$ among the total of at least $\beta\left(q / 2+\left(1-\lambda_{v}^{\prime}\right) \alpha\right)$ edges that he claimed at $v$.

Finally, each time Maker claimed an edge incident to $v$, increasing its degree in $G_{M}$ to $d+1$ from some $d$ with $d \geq \beta$, Breaker responded with at least $q / 2-(d-\beta) \alpha /(\Delta-\beta)$ edges at $v$. Hence, at all times when $v$ is large,

$$
\begin{aligned}
\operatorname{deg}_{B}(v) & \geq \frac{\operatorname{deg}_{M}(v) q}{2}+\left(1-\lambda_{v}^{\prime}\right) \alpha \beta-\sum_{d=\beta}^{\operatorname{deg}_{M}(v)-1} \frac{d-\beta}{\Delta-\beta} \alpha \\
& \geq \frac{\operatorname{deg}_{M}(v) q}{2}+\left(1-\lambda_{v}^{\prime}\right) \alpha \beta-\frac{\left(\operatorname{deg}_{M}(v)-\beta\right)^{2} \alpha}{2(\Delta-\beta)} \\
& \geq \frac{\operatorname{deg}_{M}(v) q}{2}+\left(1-\lambda_{v}^{\prime}\right) \alpha \beta-\frac{\left(\operatorname{deg}_{M}(v)-\beta\right) \alpha}{2},
\end{aligned}
$$

where the last inequality follows from the fact that $\operatorname{deg}_{M}(v) \leq \Delta$, see Claim 8. Since clearly $\operatorname{deg}_{B}(v)<n$, it follows that $\operatorname{deg}_{M}(v)<\left(2 n-\left(3-2 \lambda_{v}^{\prime}\right) \alpha \beta\right) /(q-\alpha)$.

Given a large vertex $v$, let $\lambda_{v}^{\prime}$ be as in the statement of Claim 9. Define

$$
\lambda_{v}=\frac{q / 2+\alpha-\Delta+\lambda_{v}^{\prime}(\Delta-\alpha-\beta)}{q / 2-\beta},
$$

and note that $\lambda_{v}$ is linear in $\lambda_{v}^{\prime}$.

Lemma 10. For every $t$ and every pair of vertices $u$ and $v$ such that $u v \notin G_{M}^{(t)} \cup G_{B}^{(t)}$, the following is true. Suppose $u$ and $v$ are large at the beginning of round $t+1$ and let $\lambda_{u}, \lambda_{v}$ be as defined in (6). Then for every positive $\varepsilon$, regardless of Maker's strategy,

$$
e_{B}^{(t)}(u, v) \geq \lambda_{u} \lambda_{v} \beta^{2}(q / 2-\beta) / n-\varepsilon \sqrt{n}
$$

with probability $1-e^{-\Omega_{\varepsilon}(\sqrt{n})}$.

Proof. Let $E(u, v)$ be the set of all $\operatorname{deg}_{M}^{(t)}(v)+\operatorname{deg}_{M}^{(t)}(u)$ edges (in the complete graph) from $u$ to $N_{M}^{(t)}(v)$ and from $v$ to $N_{M}^{(t)}(u)$. We start by noting that since the edge $u v$ is still unclaimed at the end of round $t$, Maker claimed no edges from $E(u, v)$, or otherwise $u v \in G_{B}^{(t)}$ (recall that Breaker immediately 'closes' all paths of length 2 that appear in $\left.G_{M}\right)$. Next, we argue that without loss of generality we may assume that random edges are the only edges in $E(u, v)$ that were claimed by Breaker. To this end, let $E_{B}^{(t)}(u, v)$ be the random variable denoting the set of all Breaker's edges in $E(u, v)$ at the end of round $t$, and let $F_{B}^{(t)}(u, v)$ be the random variable denoting the set of all such edges when we assume that Breaker did not claim any non-random edges in $E(u, v)$. Since, as we have already observed, Maker did not claim any edges from $E(u, v)$, and in the randomized procedure, given an ordering of the neighbors of a vertex, Breaker always claims those vacant edges that come first in that ordering, we always have $F_{B}^{(t)}(u, v) \subseteq E_{B}^{(t)}(u, v)$. 
Clearly, when Maker claims an edge in some round $s$, his choice can depend on the outcome of all the random decisions that Breaker made before round $s$, but it is independent of all the random decisions that Breaker made in each round $s^{\prime}$ with $s^{\prime} \geq s$ (recall that Maker moves first). Hence, the earlier Breaker has to make some random decision, the more powerful Maker becomes. Therefore, without loss of generality, we may assume that given the total of $\lambda_{u} \beta(q / 2-\beta)$ random edges to claim at the vertex $u$, Breaker was claiming the maximum possible $q / 2-\beta$ random edges after each of the first $\lambda_{u} \beta$ edges that Maker claimed at $u$, and that a similar statement holds for $v$.

Finally, we may assume that every time Maker claims an edge at $u$, the other endpoint of this edge is not incident to an edge claimed by Breaker at $v$ in earlier rounds. Similarly, Maker never claims an edge incident to $v$ whose other endpoint is already adjacent to $u$ in Breaker's graph. By following such rule, Maker can only decrease $e_{B}^{(t)}(u, v)$.

Now we are ready to estimate $e_{B}^{(t)}(u, v)$. Let $r=q / 2-\beta$ and let $p=r / n$. Since the only important rounds are those when Breaker claims random edges incident to either $u$ or $v$, we may assume that it happens precisely in the first $\left(\lambda_{u}+\lambda_{v}\right) \beta$ rounds. Also, since Breaker does not claim any random edges in later rounds, we may also assume that $t=\left(\lambda_{u}+\lambda_{v}\right) \beta$. For every $x \in\{u, v\}$ and $s$ with $0 \leq s \leq t$, let $d_{x}(s)=\operatorname{deg}_{M}^{(s)}(x)$, let $\delta_{x}(s)=d_{x}(s)-d_{x}(s-1)$, and note that by our assumptions, $d_{x}(t)=\lambda_{x} \beta$. For every $e \in E(u, v)$, let $A_{e}$ be the event that Breaker claims the edge $e$ by the end of round $t$. Assume that $e=u w$ for some $w$. By the definition of $E(u, v)$, the edge $v w$ is claimed by Maker in some round $s_{e}$ with $s_{e} \leq t$. Since Breaker claims $r\left(d_{u}(t)-d_{u}\left(s_{e}\right)\right)$ random edges at $u$ after $v w$ was claimed by Maker, then

$$
P\left(A_{e}\right) \geq \frac{r\left(d_{u}(t)-d_{u}\left(s_{e}\right)\right)}{n-1} \geq p \cdot\left(d_{u}(t)-d_{u}\left(s_{e}\right)\right)
$$

Similarly, if $e=v w$ for some $w$, and $s_{e}$ denotes the round when Maker claimed the edge $u w$, then $P\left(A_{e}\right) \geq p \cdot\left(d_{v}(t)-d_{v}\left(s_{e}\right)\right)$. Note that the map $e \mapsto s_{e}$ is a bijection between $E(u, v)$ and the set $\{1, \ldots, t\}$. Moreover, for every $u w \in E(u, v)$, Maker claims the edge $v w$ in the round $s_{u w}$, and hence $\delta_{u}\left(s_{u w}\right)=0$ and $\delta_{v}\left(s_{u w}\right)=1$. Similarly, for every $v w \in E(u, v)$, Maker claims the edge $u w$ in the round $s_{v w}$, and hence $\delta_{u}\left(s_{v w}\right)=1$ and $\delta_{v}\left(s_{v w}\right)=0$. It follows that

$$
\begin{aligned}
\mathbb{E} & {\left[e_{B}^{(t)}(u, v)\right]=\sum_{e \in E(u, v)} P\left(A_{e}\right) \geq p \cdot \sum_{s=1}^{t}\left[\left(d_{u}(t)-d_{u}(s)\right) \delta_{v}(s)+\left(d_{v}(t)-d_{v}(s)\right) \delta_{u}(s)\right] } \\
& =p \cdot \sum_{s=1}^{t}\left[\left(d_{u}(t)-d_{u}(s)\right) \delta_{v}(s)+\left(d_{v}(t)-d_{v}(s-1)\right) \delta_{u}(s)\right] \\
& =p \cdot\left[d_{u}(t) \sum_{s=1}^{t} \delta_{v}(s)+d_{v}(t) \sum_{s=1}^{t} \delta_{u}(s)-\sum_{s=1}^{t}\left(d_{v}(s) d_{u}(s)-d_{v}(s-1) d_{u}(s-1)\right)\right] \\
& =p d_{u}(t) d_{v}(t)=\lambda_{u} \lambda_{v} \beta^{2}(q / 2-\beta) / n,
\end{aligned}
$$

where the second equality follows from the fact that $d_{v}(s) \delta_{u}(s)=d_{v}(s-1) \delta_{u}(s)$. 
The following claim estimates the probability that at all times the random variable $e_{B}^{(s)}(u, v)$ does not fall much below the above lower bound on its expectation. For a positive real $\varepsilon$ and an integer $s$ with $0 \leq s \leq t$, let $B(s, \varepsilon)$ denote the event that

$$
e_{B}^{(s)}(u, v) \geq p d_{u}(s) d_{v}(s)-\varepsilon \sqrt{n}
$$

Claim 11. Let $\varepsilon, \varepsilon^{\prime}$ be positive reals and let $s, s^{\prime}$ be integers satisfying $0 \leq s \leq s^{\prime} \leq t$. If $s^{\prime}-s \leq \varepsilon \sqrt{n} /(\Delta p)$ and $\left(s^{\prime}-s\right)^{2} \leq \varepsilon^{\prime} \sqrt{n} /(2 p)$, then

$$
P\left(B\left(s^{\prime}, \varepsilon+\varepsilon^{\prime}\right) \mid B(s, \varepsilon)\right) \geq 1-\exp (-c \sqrt{n})
$$

where $c$ is a positive constant depending only on $\varepsilon^{\prime}$.

Proof. Given a $t^{\prime}$ with $0 \leq t^{\prime} \leq t$, let $e_{u}\left(t^{\prime}\right)$ and $e_{v}\left(t^{\prime}\right)$ denote the number of edges in $G_{B}^{\left(t^{\prime}\right)}$ from $u$ to $N_{M}^{(t)}(v)$ and from $v$ to $N_{M}^{(t)}(u)$, respectively. In order to estimate the conditional probability in (8), let us fix two non-negative integers $e_{u}$ and $e_{v}$ with $e_{u}+e_{v} \geq p d_{u}(s) d_{v}(s)-$ $\varepsilon \sqrt{n}$ and condition on the event that $e_{u}(s)=e_{u}$ and $e_{v}(s)=e_{v}$. Observe that at the end of round $s$, there are precisely $d_{v}(s)-e_{u}$ vertices in $N_{M}^{(s)}(v)$ that are not adjacent to $u$ in $G_{B}^{(s)}$, and there are at most $n-r d_{u}(s)$ unclaimed edges at $u$. Moreover, in rounds $s+1, \ldots, s^{\prime}$, Breaker claims $r\left(d_{u}\left(s^{\prime}\right)-d_{u}(s)\right)$ random edges at $u$, independently of $G_{M}^{(s)} \cup G_{B}^{(s)}$. Hence, under the condition $e_{u}(s)=e_{u}$, the random variable $e_{u}\left(s^{\prime}\right)-e_{u}$ is lower bounded by a random variable with distribution Hypergeometric $\left(n-r d_{u}(s), d_{v}(s)-e_{u}, r\left(d_{u}\left(s^{\prime}\right)-d_{u}(s)\right)\right)$. By Lemma 6 , with probability at least $1-\exp \left(-\left(\varepsilon^{\prime}\right)^{2} \sqrt{n} / 32\right)$,

$$
e_{u}\left(s^{\prime}\right)-e_{u} \geq \frac{\left(d_{v}(s)-e_{u}\right) r\left(d_{u}\left(s^{\prime}\right)-d_{u}(s)\right)}{n-r d_{u}(s)}-\varepsilon^{\prime} \sqrt{n} / 4
$$

If $e_{u} \leq p d_{v}(s) d_{u}(s)$, then $\left(d_{v}(s)-e_{u}\right) /\left(n-r d_{u}(s)\right) \geq d_{v}(s) / n$, and hence (9) implies that

$$
e_{u}\left(s^{\prime}\right)-e_{u} \geq p d_{v}(s)\left(d_{u}\left(s^{\prime}\right)-d_{v}(s)\right)-\varepsilon^{\prime} \sqrt{n} / 4
$$

Since a symmetric argument applies to $v$, then conditioned on the event that $e_{B}^{(s)}(u, v)=$ $e_{u}+e_{v}$, where $e_{u}+e_{v}$ is between $p d_{u}(s) d_{v}(s)-\varepsilon \sqrt{n}$ and $p d_{u}(s) d_{v}(s)$, with probability at least $1-2 \exp \left(-\left(\varepsilon^{\prime}\right)^{2} \sqrt{n} / 32\right)$, we have

$$
\begin{aligned}
e_{B}^{\left(s^{\prime}\right)}(u, v) & \geq e_{u}+e_{v}+p\left[d_{v}(s)\left(d_{u}\left(s^{\prime}\right)-d_{u}(s)\right)+d_{u}(s)\left(d_{v}\left(s^{\prime}\right)-d_{v}(s)\right)\right]-\varepsilon^{\prime} \sqrt{n} / 2 \\
& \geq p\left[d_{u}\left(s^{\prime}\right) d_{v}\left(s^{\prime}\right)-\left(d_{v}\left(s^{\prime}\right)-d_{v}(s)\right)\left(d_{u}\left(s^{\prime}\right)-d_{u}(s)\right)\right]-\left(\varepsilon+\varepsilon^{\prime} / 2\right) \sqrt{n} \\
& \geq p d_{u}\left(s^{\prime}\right) d_{v}\left(s^{\prime}\right)-p\left(s^{\prime}-s\right)^{2}-\left(\varepsilon+\varepsilon^{\prime} / 2\right) \sqrt{n} \geq p d_{u}\left(s^{\prime}\right) d_{v}\left(s^{\prime}\right)-\left(\varepsilon+\varepsilon^{\prime}\right) \sqrt{n}
\end{aligned}
$$

where the last two inequalities follow from the fact that

$$
d_{x}\left(s^{\prime}\right)-d_{x}(s) \leq d_{u}\left(s^{\prime}\right)+d_{v}\left(s^{\prime}\right)-d_{u}(s)-d_{v}(s)=s^{\prime}-s \quad \text { for } x \in\{u, v\}
$$


and the assumption that $p\left(s-s^{\prime}\right)^{2} \leq \varepsilon^{\prime} \sqrt{n} / 2$, respectively. On the other hand, if $e_{B}^{(s)}(u, v) \geq p d_{u}(s) d_{v}(s)$, then with probability 1,

$$
\begin{aligned}
e_{B}^{\left(s^{\prime}\right)}(u, v) & \geq e_{B}^{(s)}(u, v) \geq p d_{u}\left(s^{\prime}\right) d_{v}\left(s^{\prime}\right)-p\left[d_{u}\left(s^{\prime}\right) d_{v}\left(s^{\prime}\right)-d_{u}(s) d_{v}(s)\right] \\
& \geq p d_{u}\left(s^{\prime}\right) d_{v}\left(s^{\prime}\right)-p\left[d_{v}\left(s^{\prime}\right)\left(d_{u}\left(s^{\prime}\right)-d_{u}(s)\right)+d_{u}\left(s^{\prime}\right)\left(d_{v}\left(s^{\prime}\right)-d_{v}(s)\right)\right] \\
& \geq p d_{u}\left(s^{\prime}\right) d_{v}\left(s^{\prime}\right)-p \Delta\left[d_{u}\left(s^{\prime}\right)+d_{v}\left(s^{\prime}\right)-d_{u}(s)-d_{v}(s)\right] \\
& =p d_{u}\left(s^{\prime}\right) d_{v}\left(s^{\prime}\right)-p \Delta\left(s^{\prime}-s\right) \geq p d_{u}\left(s^{\prime}\right) d_{v}\left(s^{\prime}\right)-\varepsilon \sqrt{n}
\end{aligned}
$$

where the last two inequalities follow from Claim 8 (which implies that $d_{v}\left(s^{\prime}\right), d_{u}\left(s^{\prime}\right) \leq \Delta$ ) and the assumption that $p \Delta\left(s^{\prime}-s\right) \leq \varepsilon \sqrt{n}$, respectively. This completes the proof of Claim 11.

In order to finish the proof of Lemma 10 , let $K=64 / \varepsilon$ and note that

$$
K \geq \frac{16 \Delta^{2} p}{\varepsilon \sqrt{n}} \geq \max \left\{\frac{4 t^{2} p}{\varepsilon \sqrt{n}}, \frac{2 t \Delta p}{\varepsilon \sqrt{n}}\right\}
$$

For every $k \in\{0, \ldots, K\}$, let $s_{k}=k t / K$, and let $\varepsilon^{\prime}=\varepsilon /(2 K)$. By Claim 11, and the choice of $K$, see (10), for each $k$ as above, $s_{k+1}-s_{k} \leq \varepsilon \sqrt{n} /(2 \Delta p)$ and $\left(s_{k+1}-s_{k}\right)^{2} \leq \varepsilon^{\prime} \sqrt{n} /(2 p)$, and hence

$$
P\left(B\left(s_{k+1}, \frac{K+k+1}{2 K} \cdot \varepsilon\right) \mid B\left(s_{k}, \frac{K+k}{2 K} \cdot \varepsilon\right)\right) \geq 1-\exp (-c \sqrt{n}),
$$

where $c$ is a positive constant depending only on $\varepsilon$. Since clearly $B\left(s_{0}, \varepsilon / 2\right)$ holds with probability 1 , it follows that the event $B(t, \varepsilon)$, i.e., inequality $(7)$, holds with probability $1-\exp \left(-\Omega_{\varepsilon}(\sqrt{n})\right)$.

Finally, recall that in order to show that the strategy we described above actually works (i.e., prevents Maker from claiming all three edges in some triangle), it suffices to check that with nonzero probability inequality (4) is satisfied for all $u, v$, and $t$ such that $u$ and $v$ are large at time $t$. Since the number of such triples is merely polynomial in $n$, by Claim 9 and Lemma 10, it suffices to check that there is a positive $\varepsilon$ such that for all $\lambda_{u}^{\prime}$ and $\lambda_{v}^{\prime}$ in $[0,1]$, the following holds:

$$
q-\varepsilon \sqrt{n}>\frac{2 n-\left(3-2 \lambda_{u}^{\prime}\right) \alpha \beta}{q-\alpha}+\frac{2 n-\left(3-2 \lambda_{v}^{\prime}\right) \alpha \beta}{q-\alpha}-\lambda_{v} \lambda_{u} \beta^{2}(q / 2-\beta) / n .
$$

A nice property of (11) is that the right-hand side of it is linear in $\lambda_{u}^{\prime}$ and $\lambda_{v}^{\prime}$ (recall that $\lambda_{x}$ is linear in $\lambda_{x}^{\prime}$ for every vertex $x$, see (6)) and the roles of $\lambda_{u}^{\prime}$ and $\lambda_{v}^{\prime}$ are symmetric. Therefore, it is enough to check that (11) holds when the pair $\left(\lambda_{u}^{\prime}, \lambda_{v}^{\prime}\right)$ attains values $(0,0),(0,1)$, and $(1,1)$. That leaves three inequalities to check. Our choice of $q, \alpha$, and $\beta$ guarantees that all three are satisfied. 


\section{Concluding remarks}

We would like to point out that our analysis of Breaker's random strategy described in the proof of Theorem 4 leaves a lot of room for improvement. For the sake of simplicity and clarity of the presentation, in Claim 9 and Lemma 10, we settled for bounds on $\operatorname{deg}_{M}(v)$ and $e_{B}^{(t)}(u, v)$ that are fairly far from optimal. The reason for doing that was that using those weak bounds we managed to reduce the analysis of our random strategy for Breaker to checking a family of inequalities that are linear in both parameters - a task that may be easily completed by evaluating three fairly simple expressions. Below we briefly discuss possible improvements of the analysis that lead to a better bound on $q$.

The first improvement comes from observing that the argument we use in the proof of Claim 9 actually yields the following slightly stronger statement, see (5).

Claim 12. Suppose that a vertex $v$ becomes large at time $t$. Then there exists a $\lambda_{v}^{\prime} \in[0,1]$ such that $\operatorname{deg}_{M}(v)$ always satisfies

$$
n \geq \frac{\operatorname{deg}_{M}(v) q}{2}+\left(1-\lambda_{v}^{\prime}\right) \alpha \beta-\frac{\left(\operatorname{deg}_{M}(v)-\beta\right)^{2} \alpha}{2(\Delta-\beta)},
$$

and the total number of random edges that Breaker has claimed at $v$ by the end of round $t$ is at least $\beta\left(q / 2+\alpha-\Delta+\lambda_{v}^{\prime}(\Delta-\alpha-\beta)\right)$. Moreover, $\lambda_{v}^{\prime}$ depends only on what happened in the first $t$ rounds of the game.

If we solve the quadratic inequality (12) for $\operatorname{deg}_{M}(v)$, we can see that the condition on $\operatorname{deg}_{M}(v)$ in Claim 12 can be equivalently expressed in the following rather complicated form:

$$
\operatorname{deg}_{M}(v) \leq \beta+\frac{q(\Delta-\beta)}{2 \alpha}-\frac{1}{2 \alpha} \sqrt{q^{2}(\Delta-\beta)^{2}-4 \alpha(\Delta-\beta)\left[2 n-\beta q-2\left(1-\lambda_{v}^{\prime}\right) \alpha \beta\right]} .
$$

Using this stronger upper bound on the degree in (4), we can show that Breaker still wins the game $\mathbf{G}\left(K_{3} ; n, q\right)$ if $q \geq(2-1 / 22) \sqrt{n}$ (in the description of the strategy we let $\alpha=0.12 \sqrt{n}$ and $\beta=0.75 \sqrt{n})$.

Also the bound on $e_{B}^{(t)}(u, v)$ obtained in Lemma 10 can be slightly improved. In the proof of Lemma 10, we assumed that Breaker does not claim any edges at $u$ or $v$ apart from the random edges. Let us not make this assumption now and consider the particular case when Maker first claims all his $\lambda_{u} \beta$ edges at $u$ and then he claims all his $\lambda_{v} \beta$ edges at $v$. Since all the edges that Maker claimed at $v$ were chosen after Breaker had claimed all his edges at $u$, in the worst case there are no edges in $G_{B}$ that join $u$ to $N_{M}(v)$. On the other hand, $N_{M}(u)$ was a fixed set of $\lambda_{u} \beta$ vertices every time Breaker was making random choices at $v$. Moreover, the vertex $v$ was small every time Breaker claimed $q / 2-\beta$ random edges at $v$, and hence the number of unclaimed edges at $v$ reduced by at least $q / 2$. Therefore, the probability that a particular edge $e$ joining $v$ to $N_{M}(u)$ was not claimed 
by Breaker satisfies

$$
\begin{aligned}
P\left(A_{e}^{c}\right) & \leq \prod_{k=0}^{\lambda_{v} \beta-1}\left(1-\frac{q / 2-\beta}{n-k q / 2}\right) \leq \exp \left(-\sum_{k=0}^{\lambda_{v} \beta-1} \frac{q / 2-\beta}{n-k q / 2}\right) \\
& =\exp \left(-\frac{q / 2-\beta}{q / 2} \cdot \sum_{k=0}^{\lambda_{v} \beta-1} \frac{1}{2 n / q-k}\right) .
\end{aligned}
$$

Since $\log (x+1)-\log x \leq 1 / x$ for all positive $x$, it follows that

$$
\sum_{k=0}^{\lambda_{v} \beta-1} \frac{1}{2 n / q-k} \geq \log \frac{2 n}{q}-\log \left(\frac{2 n}{q}-\lambda_{v} \beta\right)-O\left(\frac{1}{\sqrt{n}}\right),
$$

and hence

$$
\mathbb{E}\left[e_{B}^{(t)}(u, v)\right]=\sum_{e \in E(u, v)} P\left(A_{e}\right) \geq \lambda_{u} \beta\left[1-\left(1-\frac{\lambda_{v} \beta q}{2 n}\right)^{1-\frac{2 \beta}{q}}\right]-o(\sqrt{n}) .
$$

Following the lines of the proof of Claim 11, one can show that with high probability,

$e_{B}^{(t)}(u, v)$ is almost as large as the above lower bound for its expectation, regardless of Maker's strategy. More precisely, the following statement can be proved.

Lemma 13. For every $t$ and every pair of vertices $u$ and $v$ such that $u v \notin G_{M}^{(t)} \cup G_{B}^{(t)}$, the following is true. Suppose $u$ and $v$ are large at the beginning of round $t+1$ and let $\lambda_{u}, \lambda_{v}$ be as defined in (6). If $\lambda_{u} \geq \lambda_{v}$, then for every positive $\varepsilon$, regardless of Maker's strategy,

$$
e_{B}^{(t)}(u, v) \geq \lambda_{u} \beta\left[1-\left(1-\frac{\lambda_{v} \beta q}{2 n}\right)^{1-\frac{2 \beta}{q}}\right]-\varepsilon \sqrt{n}
$$

with probability $1-e^{-\Omega_{\varepsilon}(\sqrt{n})}$.

Using Claim 12 and Lemma 13, we can show that Breaker still wins $\mathbf{G}\left(K_{3} ; n, q\right)$ if $q \geq 1.935 \sqrt{n}$ (in the description of the strategy we let $\alpha=0.16 \sqrt{n}$ and $\beta=0.8 \sqrt{n}$ ).

Finally, in the proof of Lemma 10, we made one other simplifying assumption. Namely, we said that for each $x \in\{u, v\}$, Breaker was claiming the maximum possible $q / 2-\beta$ random edges at $x$ after each of the first $\lambda_{x} \beta$ edges that Maker claimed at $x$. In fact, Breaker was claiming between $q / 2+\alpha-\Delta$ and $q / 2-\beta$ random edges each of the $\beta$ times when Maker claimed an edge at $x$. Therefore, inequality (13) can be further slightly improved. Unfortunately, the improvement of the lower bound on $q$ implied by such refinement of Lemma 13 is negligible (only in the third decimal place).

\section{Acknowledgement}

We would like to thank the anonymous referee for their valuable comments and suggestions. The second author would also like to thank Tomasz Luczak for introducing him to the problem. 


\section{References}

[1] J. Balogh, R. Martin, and A. Pluhár, The diameter game, Random Structures \& Algorithms 35 (2009), 369-389.

[2] J. Beck, Combinatorial games, Cambridge University Press, 2008.

[3] M. Bednarska and T. Łuczak, Biased positional games for which random strategies are nearly optimal, Combinatorica 20 (2000), 477-488.

[4] V. Chvátal and P. Erdős, Biased positional games, Annals of Discrete Mathematics 2 (1978), 221-229.

[5] H. Gebauer and T. Szabó, Asymptotic random graph intuition for the biased connectivity game, Random Structures \& Algorithms 35 (2009), 431-443.

[6] D. Hefetz, M. Krivelevich, M. Stojaković, and T. Szabó, Planarity, colorability, and minor games, SIAM Journal on Discrete Mathematics 22 (2008), 194-212.

[7] W. Hoeffding, Probability inequalities for sums of bounded random variables, Journal of the American Statistical Association 58 (1963), 13-30.

[8] M. Krivelevich, The critical bias for the Hamiltonicity game is $(1+o(1)) n / \ln n$, Journal of the American Mathematical Society 24 (2011), 125-131. 